\title{
A Multigrid Algorithm for Sampling Imaginary-Time Paths in Quantum Monte Carlo Simulations
}

\author{
C.H. Mak and Sergey Zakharov \\ Department of Chemistry, University of Southern California, \\ Los Angeles, California 90089-0482, USA
}

(Dated: December 22, 2018)

\begin{abstract}
We describe a novel simulation method that eliminates the slowing-down problem in the Monte Carlo simulations of imaginary-time path integrals near the continuum limit. This method combines a stochastic blocking procedure with the multigrid method to rapidly accelerate the sampling of paths in a quantum Monte Carlo simulation, making its dynamics more ergodic. The effectiveness and efficiency of this method are demonstrated for several one-dimensional quantum systems and compared to other standard and accelerated methods.
\end{abstract}

\section{INTRODUCTION}

The path integral formalism [1, 2] can be used to describe the statistical mechanics of a quantum system. In the path integral representation, every quantum particle maps onto a cyclic gaussian string. In most path integral simulations, the quantum strings are first discretized and then the sum over all paths is carried out by Monte Carlo sampling. In the discretized form, the path of a quantum particle is isomorphic to a classical gaussian ring polymer 3], and the statistical weight associated with each particle (in the canonical ensemble) takes the following form:

$$
W=\exp \left(-\sum_{j=1}^{P} \frac{\left|x_{j+1}-x_{j}\right|^{2}}{2 \lambda}-\epsilon V\left(x_{j}\right)\right),
$$

where $x_{j}$ is the position of the $j$-th bead on the ring, $\lambda=\epsilon \hbar^{2} / m, \epsilon=\beta / P=\left(P k_{B} T\right)^{-1}, m$ is the mass, $T$ is the temperature and the bead indices $j$ are cyclic (i.e. $P+1=1)$.

To obtain the correct quantum limit, the continuum limit $P \rightarrow \infty$ must be taken. In this limit, however, the harmonic bonds between successive beads on the ring polymer become very stiff. This causes the simulation to slow down dramatically on approach to the continuum limit, in a way that is very similar to a system undergoing a second-order phase transition. As a result, a Monte Carlo algorithm employing only local updates will equilibrate extremely slowly.

This slowing-down problem in path-integral simulations can be remedied in two ways. First, one can use a more accurate approximation for the short-time propagator in the path integral instead of the "primitive" approximation in Eqn.(1), with the hope that not too many beads will suffice to accurately approximate the path integral [4]. The second way is to devise Monte Carlo methods which employs nonlocal updates to hopefully remove or at least ameliorate the slowing-down problem.

In this paper, we are concerned with the second approach. Several alternatives to the canonical singleparticle Metropolis Monte Carlo method [5] have been proposed for this purpose. First, there is the so-called "staging" method [6, 7, 8, 9]. It attempts to reduce the correlation among the beads on the polymer by transforming to a new set of coordinates which diagonalizes the kinetic part of the action. However, in the presence of a nonzero potential $V$, the transformed coordinates become correlated to each other through $V$ again. To truncate these correlations, the staging method performs this transformation for one short segment of the ring at a time (hence the name "staging"). Updates in the transformed coordinates are done by direct sampling from independent distributions, but the new coordinates are accepted or rejected together based on a Metropolis criterion for the potential part of the action. A second method, conceptually similar to the first one, is known as the Fourier path integral method [10, 11, 12, 13]. Here the Monte Carlo moves are performed in the Fourier modes of the path, which also diagonalizes the kinetic part of the action. But the Fourier modes are also correlated through $V$. A third method, which is also based on similar ideas as the first two, is the bisection method [4, 14]. Instead of generating the Fourier modes of the path, the bisection method samples the midpoint of a large segment of a free-particle path and accept or reject it using an approximation for the long-time action that includes effects of the potential. If the midpoint is accepted, the midpoints of the two shorter subsegments on each side of the midpoint are then generated, and this process is iterated until every bead on the entire segment is generated. Finally, there is a multigrid-based method, first applied to path integrals by Janke and Sauer [15, 16. They propose moving whole blocks of neighboring beads on multiple length scales using a Metropolis algorithm, and they cycle through the different length scales in a systematic way.

In this paper, we describe another Monte Carlo method. This method is actually related to the four methods described above, but as we will show, its formulation is more general, its applications are more powerful and its efficiency is much higher than the previous methods. Our method combines a stochastic blocking procedure, often referred to as the Swendson-Wang method [17], with multigrid ideas 18, 19] in an attempt to formulate a set of equilibrium stochastic dynamics that is 
highly ergodic for path integral simulations. The idea for this type of multigrid method was first proposed by Kandel et al. for an Ising model at criticality 20].

The method will be described in Sections IIIII Section III provides the general concept of the method, and Section III applies the concept to the path integral problem. Section IV will compare the method against others for several examples of 1-dimensional systems with single- and double-well potentials.

\section{GENERAL FORMULATION}

The general idea of the multigrid Monte Carlo method has been described in detail by Kandel et al. [20] for the Ising model. We will not attempt to reproduce all the details here. Instead, we will summarize the essentials in this section, using a language which is closer to path integrals. The specific application of these ideas to path integral simulations will be described in detail in Sect. III

Consider a system with partition function

$$
Q=\int d x_{1} \cdots d x_{N} e^{-\mathcal{S}},
$$

where $\mathcal{S}=\sum_{\alpha} u_{\alpha}$, and each $u_{\alpha}$ is a real-valued interaction term involving any number of the $N$ particles in the system. Of course, all classical systems, as well as quantum systems that can be mapped onto isomorphic classical polymeric systems through the path integral formalism, have partition functions of this form. The correlations among the particles arise from the interactions $u_{\alpha}$.

The multigrid Monte Carlo method is based on a combination of the stochastic blocking and multigrid ideas. We will first describe the stochastic blocking procedure, often referred to as the "unigrid" method. To accelerate the dynamics of the system, the unigrid method proceeds in two stages. First, with the current configuration $X=\left\{x_{1}, \cdots x_{N}\right\}$, we attempt to remove some of the correlations from among the particles by "killing" the interaction terms $u_{\alpha}$ one by one: For each $u_{\alpha}$, we consider either "deleting" it entirely from the action $\mathcal{S}$ with probability $p_{d}=c_{\alpha} \exp \left(u_{\alpha}\right)$ or "freezing" it with probability $p_{f}=1-p_{d}$. If an interaction is deleted, the ensuring simulation can update $X \rightarrow X^{\prime}$ without any regard to $u_{\alpha}$. If on the other hand $u_{\alpha}$ is frozen, the ensuring simulation must not change the value of $u_{\alpha}$ during any update $X \rightarrow X^{\prime}$. To ensure that $p_{d}$ and $p_{f} \in[0,1]$, the coefficient $c_{\alpha}$ must be chosen to be smaller than $\exp \left(-u_{\alpha}^{*}\right)$, where $u_{\alpha}^{*}$ is the largest possible value for $u_{\alpha}$. After all the interactions have been killed (deleted or frozen), the particles can be divided into separate clusters - particles in the same cluster are connected by frozen bonds, while particles in different clusters are no longer correlated with each other.

In the second stage of the simulation, we can update each cluster separately with a Monte Carlo move that preserves the frozen bonds inside that cluster. After all the clusters have been updated, we can restore the interaction terms and repeat the procedure starting from the first stage again, or we can use a few local Metropolis moves to update the system before starting the stochastic blocking procedure again. It can easily be shown that this two-stage procedure satisfies detailed balance and therefore produces the correct statistical sampling [17].

Under the unigrid method, interactions that are strong will more likely be frozen and those that are weak will more likely be deleted. This operation aims to remove some of the interactions from the system and this can potentially make the subsequent updates more ergodic. Whether this is actually the case will depend on two factors: (1) whether the length scale of the unconnected clusters are actually small enough, and (2) whether there exists an efficient way to update each cluster without disturbing the frozen interactions. In reality, the length scale of unconnected clusters resulting from the stochastic blocking procedure can still be quite large, and hence a lot of the correlations remain in the system. In addition, for systems with continuous coordinates, finding an efficient way to update all the particles in an unconnected cluster while preserving the frozen interactions is not always trivial. Therefore, the stochastic blocking procedure ends up not being as useful as it may appear.

To repair this and to completely remove the residual correlations, we incorporate multigrid ideas and try to force the clusters to break up into smaller pieces of varying length scales. To achieve this, the multigrid method first divides the particles into sets, each having a different length scale. In this context, the definition of the "length scale" should be based on an intuitive understanding of the physical origin of the correlations in the system. (For example, in path integrals, the dominant correlations among the beads on a ring originate from the harmonic bonds, so these correlations can be decomposed into a hierarchy of gaussian fluctuations on different length scales.) After a definition of these sets is made, we proceed as before but with the stochastic blocking procedure applied to only particles belonging to a single length scale. As such, we kill all the interactions between particles of that length scale, while the other interactions among particles on all other length scales are kept alive. The result of the blocking procedure produces clusters that are unconnected by interactions on the current length scale, but these "unconnected" clusters are not totally independent because they are still correlated with each other through the interactions that are kept alive. We update the unconnected clusters as before, but to maintain detailed balance, each update will also have to be accepted or rejected using a Metropolis criterion based on all the live interactions that are linked to that cluster. After updating all the "unconnected" clusters on one length scale, we can proceed to another one. In this manner, the multigrid method systematically breaks up all the remaining correlations on every length scale and the slowing-down problem can be completely eliminated. It can also be shown that this multigrid procedure satis- 
fies detailed balance and therefore produces the correct statistical sampling [20].

\section{APPLICATION TO PATH INTEGRAL SIMULATIONS}

In this section, we discuss the application of the method in Sect. II to path integral simulations. Here, we assume a 1-dimensional particle in a potential $V$. Generalization to higher dimensions and many particles is straightforward.

\section{A. Definition of Length Scales}

Before describing the unigrid and multigrid algorithms, we define the concept of length scales in a path integral simulation. Let the number of beads on the ring $P$ be equal to $2^{L}$, where $L$ is a positive integer. (Since the path is cyclic, bead 0 is identical to bead $2^{L}$.) We divide the beads into different "levels" $\ell=0,1, \cdots L$, such that $\ell=\left\{1 \times 2^{\ell}, 3 \times 2^{\ell}, 5 \times 2^{\ell}, \cdots\right\}$. For examples, $\{1,3,5, \cdots\}$ would belong to $\ell=0,\{2,6,10, \cdots\}$ to $\ell=1$, $\{4,12,20, \cdots\}$ to $\ell=2$, etc.

Using these definitions, we say the full path is of length scale $L$. Starting with the full path, we divide it into segments of different length scales at specific endpoints. Bisecting the full path yields two equal-length segments of length scale $L-1$, the first one having endpoints 0 and $2^{L-1}$ and the second $2^{L-1}$ and $2^{L}$. Bisecting each of these $L-1$ length scale segments again, we get four segments of length scale $L-2$, and so on.

In the absence of a potential $V$, every path segment of every length scale can be sampled independently from a gaussian distribution. Therefore, even though beads on different levels $\ell$ are connected with each other via the kinetic energy springs, the path segments on all length scales can actually be generated in a completely uncorrelated manner. But the presence of a nonzero potential $V$ introduces correlations back into the path segments, and they can no longer be sampled independently. The potential produces a "confinement" effect on the path, which couples path segments of certain length scales. The length scale of these correlations depends on the spatial extent of the confining potential as well as the temperature. For a fixed temperature, a broad and shallow potential produces less correlation than a steep narrow potential. More complicated potentials (those frequently present in condensed systems) may produce correlations on multiple length scales. For example, a bistable potential produces two length scales, one for intra-well quantum fluctuations and the other for inter-well fluctuations. The goal of the stochastic blocking method is to remove some of these correlations produced by the potential, and the multigrid method furthermore refines it by attempting to remove these correlations over all length scales.

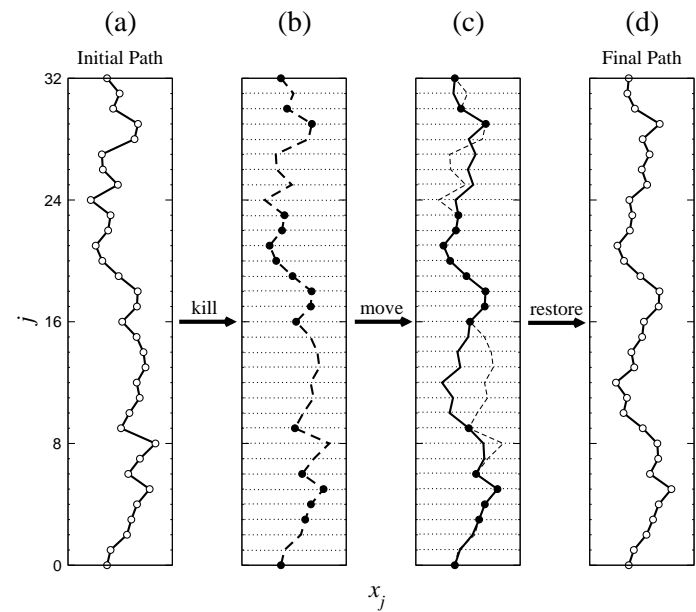

FIG. 1: Illustration of the unigrid stochastic blocking procedure. (See text for details.)

\section{B. Stochastic Blocking: The Unigrid Method}

The stochastic blocking method aims at killing the correlations that come from the potential terms $V\left(x_{j}\right)$ in Eqn.(11). Following the ideas described in Sect. [I] we kill every $V\left(x_{j}\right)$ by either deleting it or freezing it. This is illustrated in Fig. 1 for a path with $L=5$. Panel (a) shows the original path, with the $V\left(x_{j}\right)$ on all beads $i$ depicted pictorially as open circles. The stochastic blocking is done by first freezing the endpoints of the full path at $j=0=2^{L}$ and then killing every potential term $V\left(x_{j}\right)$ for $0<j<2^{L}$. This operation is represented in panel (b). The dotted lines highlight the beads on which the killing operation is performed, and the result of each killing operation is either a frozen bead (represented by a closed circle) or a deleted bead (represented by the absence of a circle). The path breaks up into frozen segments consisting of beads $0(=32), 3-6,9,16-23$ and 29-30 and the intervening deleted segments. The frozen segments can not be moved, but the deleted segments can be sampled independently from gaussian distributions for free-particle paths of various lengths. Panel (c) shows the new path after the move (solid line) compared to the initial path (dashed line). All the potential terms $V\left(x_{j}\right)$ are finally restored resulting in the final path in panel (d). This completes one pass and the next pass begins anew with the killing procedure performed on the path in panel $(d)$. (Since the origin of the cyclic path at $j=0=2^{L}$ is always frozen in this method, we select a different origin at random on every pass to maintain ergodicity.)

Before moving on to the multigrid implementation, we want to mention one minor difference between what is described here and the original implementation of the stochastic blocking procedure due to Swendson and Wang 17] as summarized in Sect. [I] The stochastic blocking procedure was originally applied to the Ising model, a system where the state of each particle belongs 

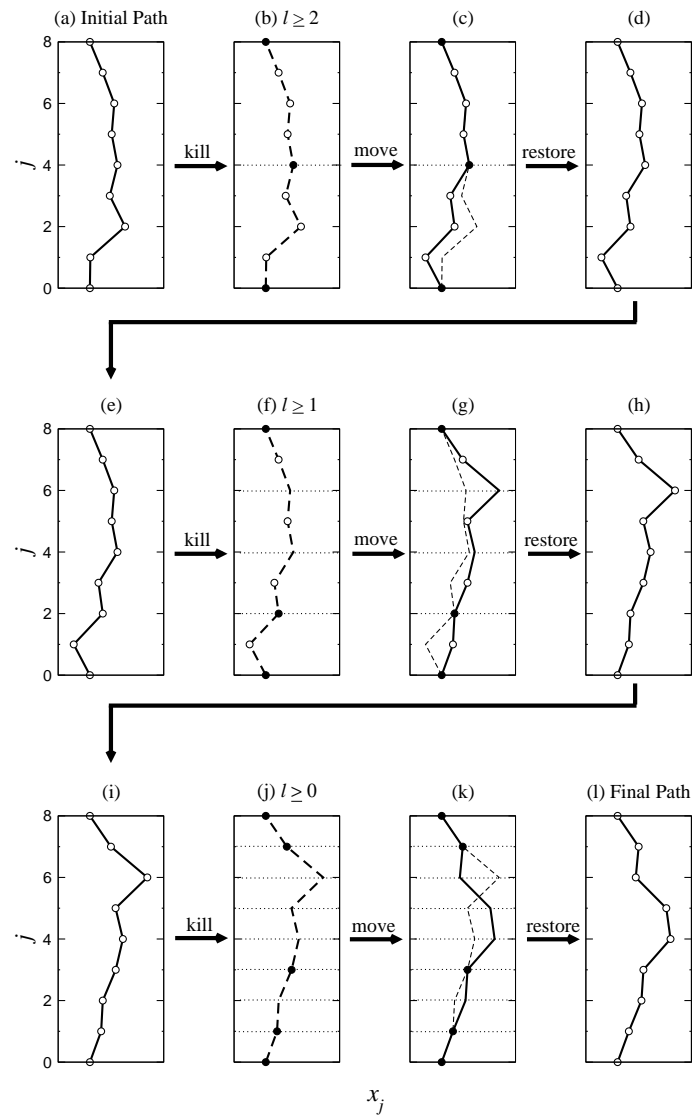

FIG. 2: Illustration of the multigrid procedure. (See text for details.)

to a finite discrete set and every potential term $u_{\alpha}$ in the action of the Ising model is bounded from above by $u_{\alpha}^{*}$. As discussed in Sect. III to ensure that the deletion probabilities $p_{d} \in[0,1], c_{\alpha}$ can be chosen to be less than $\exp \left(-u_{\alpha}^{*}\right)$ for every potential term. In our path integral application, however, the coordinate of each bead is a continuous variable and the potential is in general not bounded from above. This means that to strictly satisfy the requirement that $p_{d} \leq 1, c_{\alpha}$ must be chosen to be 0 which then results in no deletion at all. In practice, we can circumvent this minor problem by choosing a sufficiently large $u_{\alpha}^{*}$ and monitors the frequency at which the bound $p_{d} \leq 1$ is violated during the simulation. By adjusting $u_{\alpha}^{*}$ to yield a bound violation frequency of less than $0.01 \%$, we can maintain a relatively high deletion ratio while introducing negligible errors to the results.

\section{The Multigrid Method}

The multigrid method makes use of the stochastic blocking procedure in the unigrid method, but forces the path segments to break up on a predefined set of length scales. The procedure is illustrated pictorially for a path with $L=3$ in Fig. 2
Starting with the initial path in panel (a), we kill all $V\left(x_{j}\right)$ on levels $\ell \geq 2$. This results in a frozen bead at $j=$ 4. The open circles in panel (b) indicate $V\left(x_{j}\right)$ that are kept alive. When the new path segments are generated, they must be accepted or rejected based on a Metropolis criterion involving all the live beads. Panel (c) shows the new path after the move: the new segment between $j=0$ and 4 is accepted based on the three live beads at $j=1-3$, but the other new segment between $j=4$ and 8 is rejected based on the three live beads at $j=5-7$; as a result, the new path (solid line) between $j=4$ and 8 coincides with and the old one (dashed line). Panel (d) shows the new path on this level after all $V\left(x_{j}\right)$ are restored. Then we move on to the next finer level. On this level, we kill all beads on level $\ell \geq 1$, namely at $j=2,4$ and 6 . This results in the frozen (solid circles), deleted (no circles) and live (open circles) beads indicated in panel (f). Two new segments are generated independently and accepted based on the live bonds at $j=1$ and $j=3,5$ and 7 . All beads are then restored and the procedure repeats on the finest level in the bottom row of Fig. 2] starting with killing all $V\left(x_{j}\right)$ for $\ell \geq 0$.

Notice that the method presented here is very different from a method previously described by Janke and Sauer [15, 16], which they also call a "multigrid path integral method".

\section{RESULTS}

We have carried out numerical tests on our algorithm for several 1-dimensional quantum systems and compared them against other methods, including:

1. Metropolis: a conventional Metropolis algorithm based on single-bead moves in the $x$-coordinates;

2. Bisection: the bisection algorithm as described by Ceperley in [4];

3. Unigrid: the unigrid algorithm as described above; and

4. The so-called "multigrid path integral method" of Janke and Sauer [15, 16].

The tests were carried out on different model systems with symmetric single- and double-well potentials. In the simulations, we have adopted a system of dimensionless units in which $m=\hbar=1$. For all tests, $\beta=10$, yielding a thermal wavelength of approximately 1.6 which sets the length scale of the quantum dispersion due to the kinetic part of the action. For all of the systems studied, the ground state dominates at this temperature. Because the potentials are all symmetric, $\langle x\rangle$ should be 0 ; therefore, how quickly the measured $\langle x\rangle$ goes to the exact value of 0 will provide a good estimate of the efficiency of each algorithm. Alternatively, if we calculate the quantity $\bar{x} \equiv$ $\sum_{j=1}^{P} x_{j}$ after each Monte Carlo step, we can determine the efficiency of the algorithm by examining how rapidly $\bar{x}$ fluctuates around the exact value $\langle x\rangle=0$. 


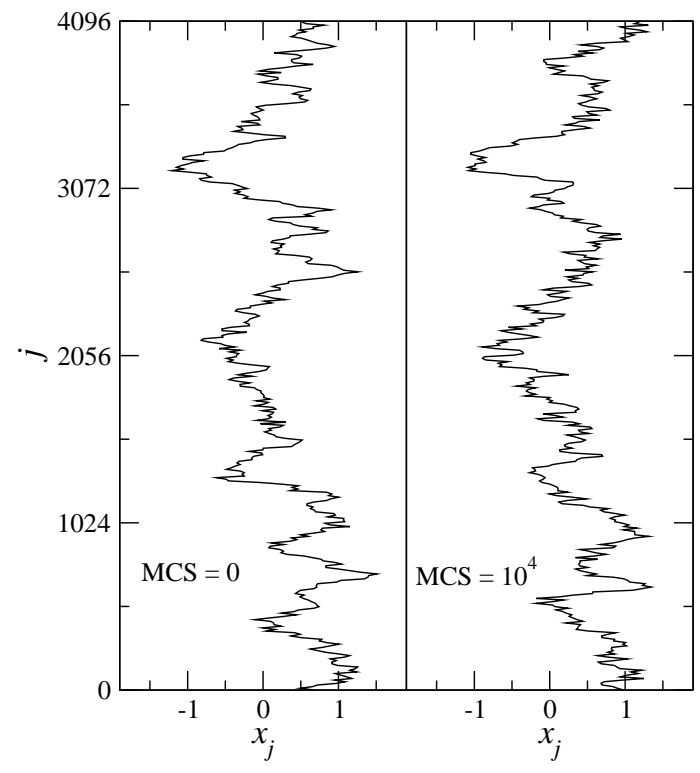

FIG. 3: Initial and final paths after $10^{4}$ MCS in a conventional Metropolis Monte Carlo simulation of Model A using a discretization of $L=12$. The configuration has hardly moved, indicating the severity of the slowing-down problem.

\section{A. Harmonic Potential (Model A): $V(x)=\frac{1}{2} x^{2}$}

Model A is a simple harmonic potential. Figure 3 shows the initial and final quantum paths after $10^{4}$ singleparticle Metropolis Monte Carlo steps (one MCS is defined as having every bead on the path subjected to one trial move on the average). There is very little movement in the configuration of the whole path. Clearly, the conventional Metropolis algorithm is highly ineffective. $P=4096$ beads, or $L=12$, were used to represent the path here. With $P$ of this magnitude, the bead-tobead dispersion is merely 0.05 , roughly $3 \%$ of the thermal wavelength, making the harmonic bonds between successive beads along the ring extremely stiff.

The discretization $L=12$ is much larger than what is needed for the path integral results to converge to the continuum limit for this model. The minimum required $L$ is 5 . Figure 4 shows $\bar{x}$ as a function of MCS for the four methods for $L=5$. Visually, we can see that the multigrid method is the most efficient, the unigrid and the bisection methods are comparable and slower than the multigrid method, and the Metropolis method is the least efficient. To get a more precise measure of the efficiencies, the data in Fig. 4 were autocorrelated and the correlation functions are shown in Fig. 5 for the four methods. The general conclusions we obtained from a visual inspection of Fig. 4 are confirmed by Fig. 15 - the multigrid method has the fastest decay time constant, approximately $1 \mathrm{MCS}$, and therefore is the most efficient.

The efficiencies of the four different methods for other values of $L$ are shown in Fig. [6 The left panel of Fig. 6 shows the decay time constants $\tau_{c}$ in units of MCS from

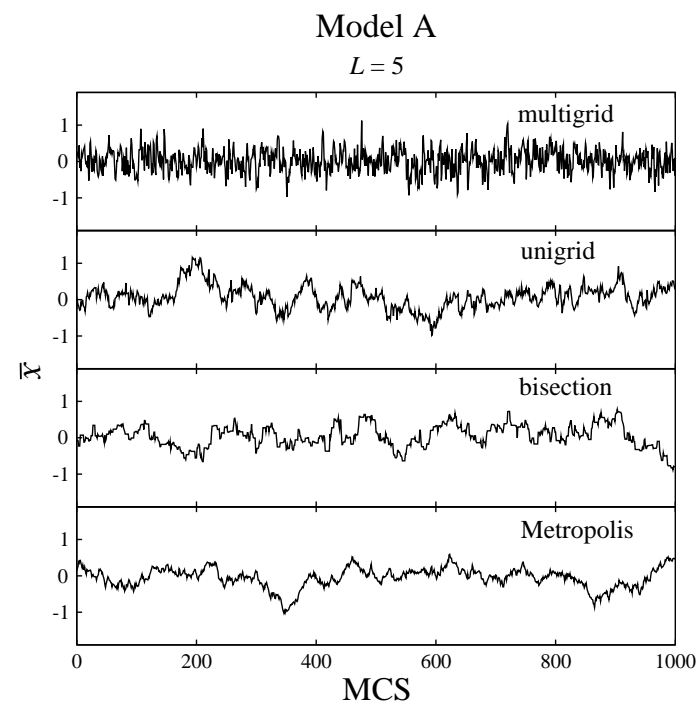

F

of

ti

tr

fll

ce

$\mathrm{p}$

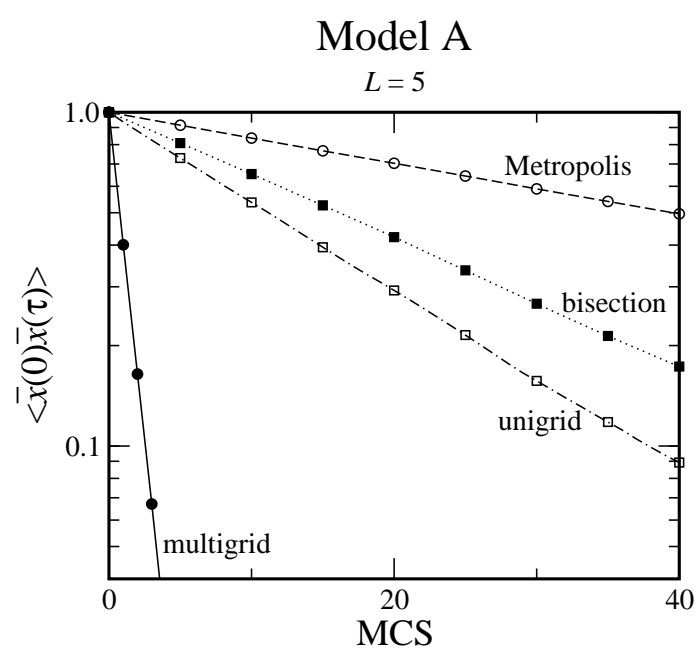

FIG. 5: Autocorrelation functions of $\bar{x}$ for the data in Fig. 4 The multigrid method has the fastest decay time.

the autocorrelation function of $\bar{x}$ for the four methods at different path discretizations $L$. The vertical line at $L=$ 5 indicates the minimum value of $L$ needed for the path integral results to converge for this model. Everything on the left of this line does not converge to the correct continuum limit. Therefore, the only relevant results are those to the right of this line.

From a computational standpoint, the ultimate measure of real efficiency is the correlation time measured in actual $C P U$ cycles (i.e. $\tau_{c}$ in units of MCS multiplied by CPU time per MCS) since difference algorithms will 
Model A

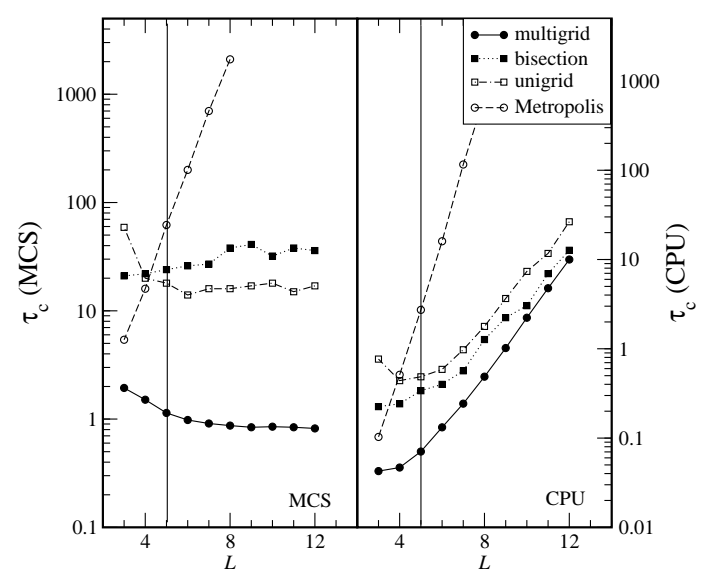

FIG. 6: Correlation times $\tau_{c}$ from four different MC methods at different discretizations $L$ for Model A. The vertical line at $L=5$ indicates the minimum $L$ needed for the path integral results to converge to the continuum limit. The left panel shows correlation times in units of MCS. The right panel shows the same but in units of actual CPU millisecond. (The segment length used for the bisection method was $2^{L-2}$ for each L.)

incur different CPU time per MCS. The right panel in Fig. [6] shows correlation times in actual CPU milliseconds. The real efficiency of the multigrid method at the minimum required discretization for convergence $(L=5)$ is about a factor of 4 better than its nearest competitor, the bisection method, and a factor of 40 better than the standard Metropolis method.

This model has essentially one length scale dictated by the confinement effect of the potential. For the particular parameters chosen for this model, the confinement length scale of the potential turns out to be quite similar to the natural thermal wavelength of the free-particle path. For this situation, a relatively small $L$ is sufficient for the path integral to converge to its continuum limit. Moreover, the four different methods, with their correlation times spanning only one and a half orders of magnitude, do not exhibit vastly different efficiencies.

These conclusions drawn from Model A are certainly not universal. The relative efficiencies of the various methods will depend on a number of factors, such as the length scale of the potential and the temperature. The additional examples presented below demonstrate this. But in all the models considered, the multigrid method was always the most efficient by an order of magnitude or more compared to any other method.

\section{B. Compressed Harmonic Potential (Model B): $V(x)=\frac{1}{2}(3 x)^{2}$}

In Model B, the length scale of the potential is substantially smaller than the thermal wavelength. This sit-

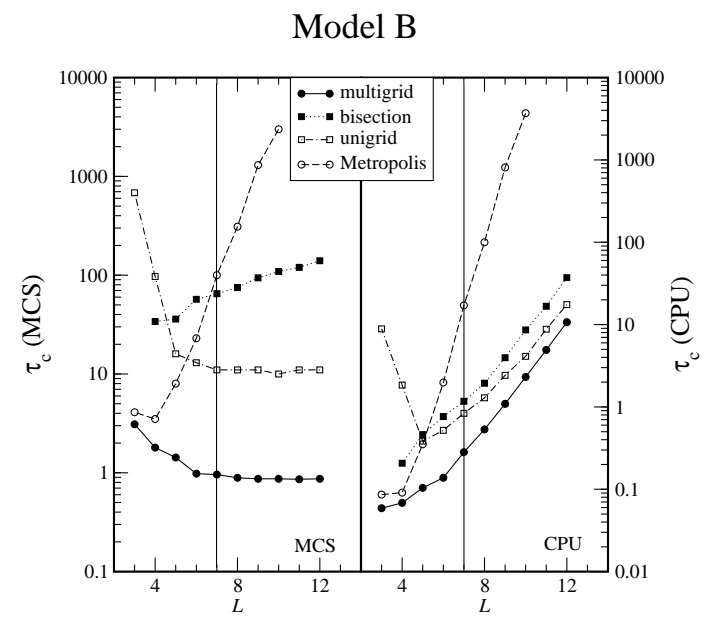

FIG. 7: Correlation times $\tau_{c}$ from four different MC methods at different discretizations $L$ for Model B. The minimum discretization required for convergence is $L=7$, indicated by the vertical line. Left and right panels show $\tau_{c}$ in units of MCS and CPU millisecond, respectively. (The segment length used for the bisection method was $2^{L-3}$ for each $L$.)

uation is quite typical in real condensed-phase quantum systems. The decay time constants of the autocorrelation functions of $\bar{x}$ are summarized in Fig. 7 for different $L$ and the four MC methods. For this model, $L=7$ is the minimum required for convergent path integral results. At or above this value of $L$, the multigrid method outperforms all the other methods in both MCS and CPU efficiencies. For this model, the second most efficient method is the unigrid method, which performs at about a factor of 4 poorer than the multigrid method at $L=7$ in terms of real CPU efficiency. On the other hand, the Metropolis method is a factor of 80 less efficient.

\section{Double-Well Potential (Model C): $V(x)=-3 x^{2}+x^{4}$}

Model $\mathrm{C}$ is a double-well potential with a moderate barrier. The inter-well separation is somewhat longer than the thermal wavelength but not by much. This is the first model that has at least two length scales due to the bistable nature of the potential. Because of the moderate barrier, the length scales of the intra-well and inter-well quantum fluctuations are in reality not very different from each other.

The decay time constants of the autocorrelation functions of $\bar{x}$ are summarized in Fig. 8 for different $L$ and the four MC methods. For this model, $L=7$ is the minimum required for convergent path integral results. At or above this value of $L$, the multigrid method outperforms all the other methods in both MCS and CPU efficiencies. For this model, the next most efficient method is the bisection method, which performs at about a factor of 3 poorer than the multigrid method at $L=7$ in terms of 


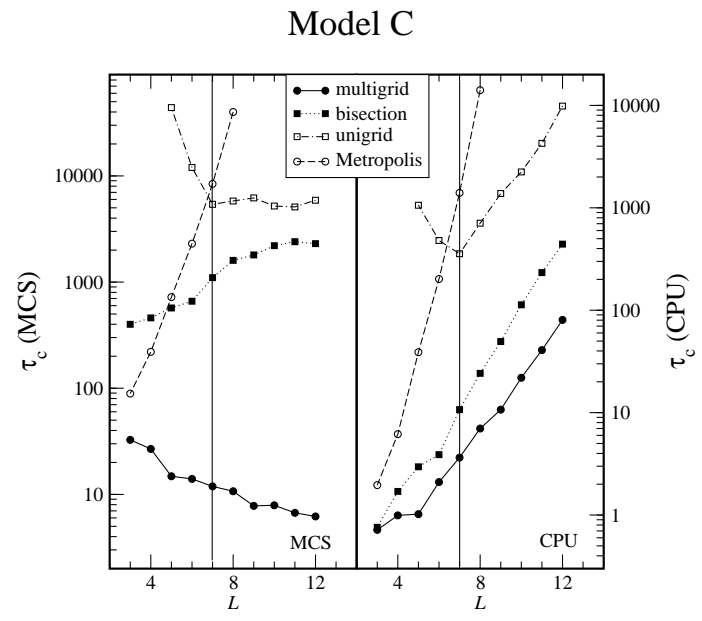

FIG. 8: Correlation times $\tau_{c}$ from four different MC methods at different discretizations $L$ for Model C. The minimum discretization required for convergence is $L=7$, indicated by the vertical line. Left and right panels show $\tau_{c}$ in units of MCS and CPU millisecond, respectively. (The segment length used for the bisection method was $2^{L-2}$ for each $L$.)

real CPU efficiency.

\section{Compressed Double-Well Potential (Model D): $V(x)=-3(2 x)^{2}+(2 x)^{4}$}

Model D has the same double-well potential as Model $\mathrm{C}$, but the potential is compressed in the $x$ direction. In addition to having two distinct length scales, this model is further complicated by having a rather severe confinement effect because the length scale of the potential is much smaller than the natural thermal wavelength of the free-particle path. The result is that the minimum discretization required for convergence becomes larger $(L=8)$ and the multigrid method becomes increasingly advantageous compared to the other methods. On the other hand, the bisection method suffers here, because it generates path segment of only one predefined length scale. When that length scale is adjusted for maximum optimal efficiency, it would match one but misses all the other relevant length scales present in the problem 21].

The decay time constants of the autocorrelation functions of $\bar{x}$ are summarized in Fig. 9 for different $L$ and three MC methods. The results from the unigrid method are not shown because for every $L$ the decay time in the unigrid results is greater than $10^{4} \mathrm{MCS}$ and we were unable to equilibrate the unigrid simulations. The multigrid method outperforms the other two methods in both MCS and CPU efficiencies. For this model, the next most efficient method is the bisection method, which performs at about a factor of 10 poorer than the multigrid method at $L=8$ in terms of real CPU efficiency.

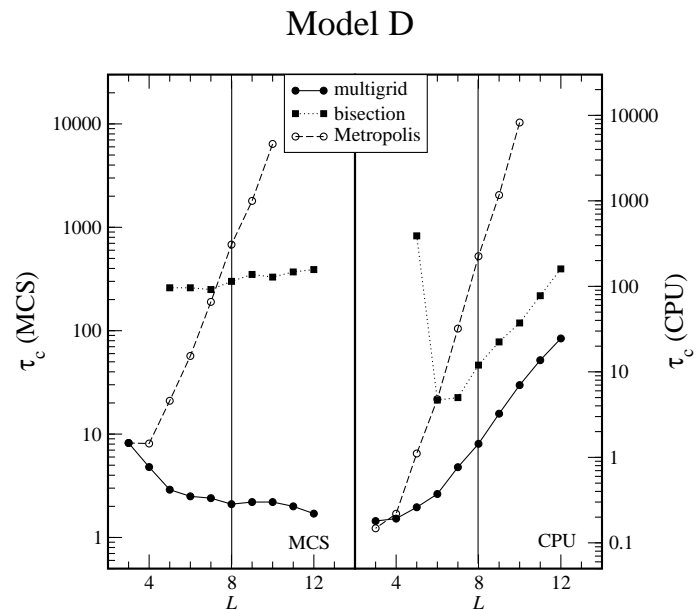

FIG. 9: Correlation times $\tau_{c}$ from three different MC methods at different discretizations $L$ for Model D. The minimum discretization required for convergence is $L=8$, indicated by the vertical line. Left and right panels show $\tau_{c}$ in units of MCS and CPU millisecond, respectively. (The segment length used for the bisection method was $2^{L-4}$ for each $L$.)

\section{E. Model of Janke and Sauer (Model E): $V(x)=-0.5 x^{2}+0.04 x^{4}$}

To make contact with the results of Janke and Sauer [15, 16], we carried out simulations on the double-well potential studied in their papers, using the same parameters they have used. The decay time constants of the autocorrelation functions of $\bar{x}$ are summarized in Fig. 10 for different $L . L=7$ is the minimum required for convergent path integral results. The Metropolis, unigrid and bisection results are not shown because their decay times are all greater than $10^{4}$ MCS for all values of $L$. At $L=7$, the multigrid method is about an order of magnitude more efficient than the Janke/Sauer method. But notice that the CPU correlation time for this model is much larger $\left(\tau_{c} \approx 100 \mathrm{CPU} \mathrm{ms}\right)$ compared to all the previous models. Therefore, this model potential seems to present a slightly more challenging problem even for the multigrid method.

\section{F. An Electron in the Field of Two Positive Ions (Model F)}

This last model consists of a 1-dimensional electron in the field of two ions of +2 charge separated by a distance of $15 \AA$. To prevent the electron path from collapsing onto either ion, we set up a $0.25 \AA$ hard core radius around each. The temperature is $300 \mathrm{~K}$ and the dielectric constant is 78. Similar to some of the other models already considered, this model consists of a bistable potential with a high barrier between the two wells. This model is however qualitative quite different from the others, because the coulombic potential centered on each ion 


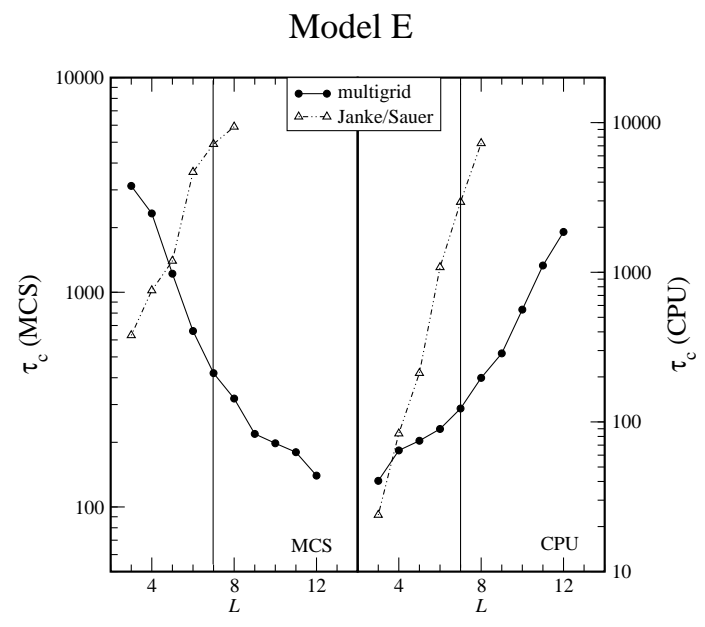

FIG. 10: Correlation times $\tau_{c}$ from three different MC methods at different discretizations $L$ for Model E. The minimum discretization required for convergence is $L=7$, indicated by the vertical line. Left and right panels show $\tau_{c}$ in units of MCS and CPI millisecond. resnectivelv.

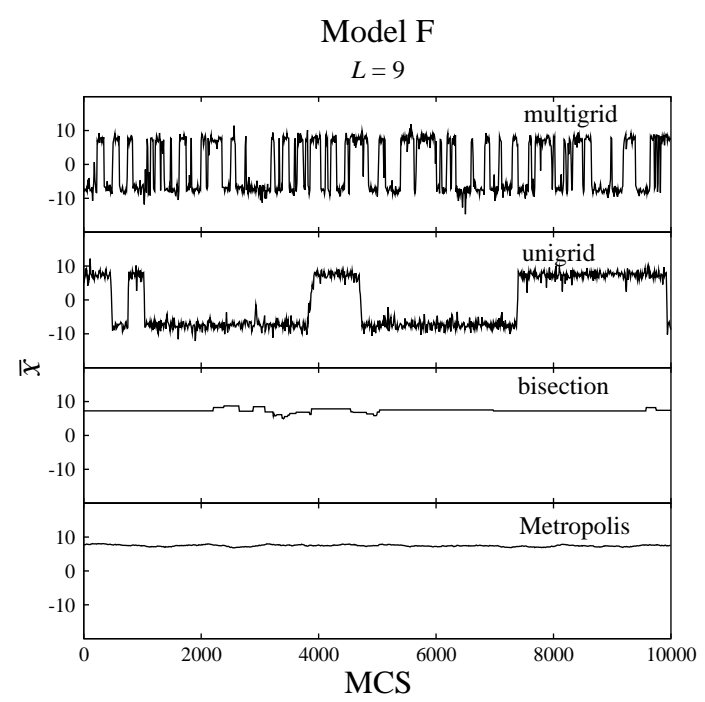

FIG. 11: Measurement of $\bar{x}$ after each MCS during the course of the MC simulation for Model F using the Metropolis, the bisection, the unigrid and the multigrid methods using a discretization of $L=9$.

is much narrower in comparison with the fairly open well bottoms in the other models. This results in a strong confinement effect on the electron path. The natural quantum fluctuations of the path are of a longer scale than the width of the potential wells. Figure 11 shows $\bar{x}$ as a function of MCS for the four methods at $L=9$, the minimum required discretization needed for convergence.
Both the bisection and the Metropolis methods failed to equilibrium in 10000 MCS. The decay time constants of the autocorrelation function of $\bar{x}$ are shown in Fig. 12 for the

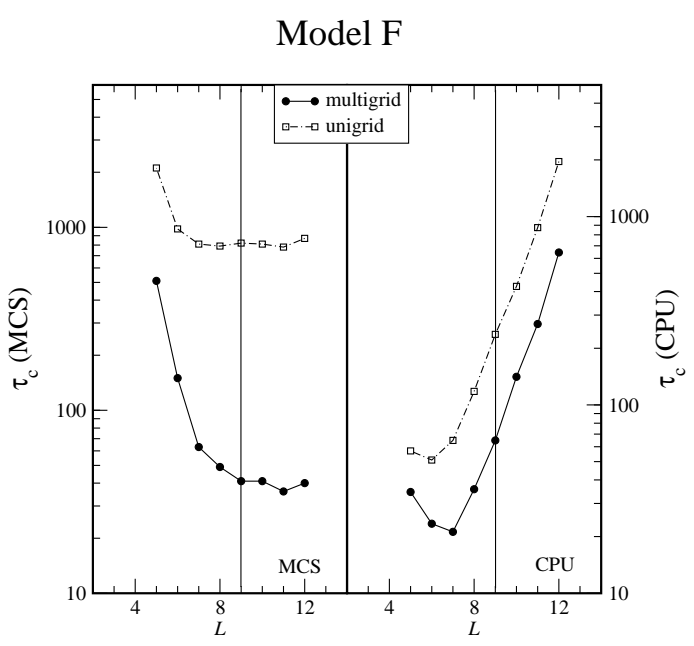

FIG. 12: Correlation times $\tau_{c}$ from the multigrid and the unigrid methods for Model F. The minimum discretization required for convergence is $L=9$, indicated by the vertical line. Left and right panels show $\tau_{c}$ in units of MCS and CPU millisecond, respectively.

\section{CONCLUSION}

We have described a new Monte Carlo method for simulating imaginary-time path integrals. The new method uses a combination of a stochastic blocking algorithm and multigrid ideas to completely eliminate the slowing-down problem in the sampling of discretized quantum paths near the continuum limit. The method has been tested on several 1-dimensional quantum systems and found to exhibit highly ergodic dynamics. The new method offers distinct advantages over other methods in cases where the length scale of the potential is smaller than the quantum dispersion of the path, a situation that is quite typical in real condensed-phase quantum systems. On the other hand, for systems with bistable potentials with length scales much larger than the quantum dispersion of the path, the new method, though better than all the other methods, has only limited utility.

\section{ACKNOWLEDGMENTS}

This work was supported by the National Science Foundation (CHE-9970766).
[1] Feynman, R.P.; Hibbs, A.R. Quantum Mechanics and Path Integrals; McGraw-Hill: New York, 1965.
[2] Feynman, R.P. Statistical Mechanics; Benjamin/Cummings: Reading, 1972. 
[3] Chandler, D.; Wolynes, P.G. J. Chem. Phys. 1981, 74, 4078.

[4] Ceperley, D.M. Rev. Mod. Phys. 1995, 67, 279.

[5] Metropolis, N.; Rosenbluth, A.W.; Rosenbluth, M.N.; Teller, A.H.; Teller, E. J. Chem. Phys. 1953, 21, 1087.

[6] Pollock, E.L.; Ceperley, D.M. Phys. Rev. B 1984, 30, 2555.

[7] Sprik, M.; Klein, M.L.; Chandler, D. Phys. Rev. B 1985, 31, 4234.

[8] Sprik, M.; Klein, M.L.; Chandler, D. Phys. Rev. B 1985, $32,545$.

[9] Tuckerman, M.E.; Berne, B.J.; Martyna, G.J.; Klein, M.L. J. Chem. Phys. 1993, 99, 2796.

[10] Doll, J.D. J. Chem. Phys. 1984, 81, 3536.

[11] Freeman, D.L.; Coalson, R.D.; Doll, J.D. J. Stat. Phys. 1986, 43,931 .

[12] Lobaugh, J.; Voth, G.A. J. Chem. Phys. 1992, 9\%, 4205.
[13] Mielke, S.L.; Truhlar, D.G. J. Chem. Phys. 2001, 114, 621.

[14] Chakravarty, C.; Gordillo, M.C.; Ceperley, D.M. J. Chem. Phys. 1998, 109, 2123.

[15] Janke, W.; Sauer, T. Chem. Phys. Lett. , 201L499, 505.

[16] Janke, W.; Sauer, T. Chem. Phys. Lett. 1996, 263, 488.

[17] Swendsen, R.H.; Wang, J.-S. Phys. Rev. Lett. 1987, 58, 86.

[18] Briggs, W.L. Multigrid Tutorial; Society for Industrial and Applied Mathematics: Philadelphia, 1987.

[19] Goodman, J.; Sokal, A.D. Phys. Rev. D 1989, 40, 2035.

[20] Kandel, D.; Domany, E.; Ron, D.; Brandt, A.; Loh, E.,Jr. Phys. Rev. Lett. 1988, 60, 1591.

[21] It may be possible that the efficiency of the bisection method can be improved by incorporating a multigrid element into it. 